\title{
Power and Flow Assignment for 5G Integrated Terrestrial-Satellite Backhaul Networks
}

\author{
Eva Lagunas*, Lei Lei, Symeon Chatzinotas, Björn Ottersten \\ Interdisciplinary Centre for Security, Reliability and Trust (SnT), University of Luxembourg \\ Email: \{eva.lagunas,lei.lei,symeon.chatzinotas,bjorn.ottersten\}@uni.lu \\ ${ }^{*}$ Corresponding author
}

\begin{abstract}
The optimal flow assignment is strongly dependent on the network link capacities, which in turn are determined by the allocation of the available radio resources. In this paper, we consider the holistic design of joint power and flow assignment in the context of Integrated Terrestrial-Satellite Backhaul (ITSB) networks. Aiming for an spectral efficient system, we focus on the scenario where the satellite links operate in the nonexclusive Ka band, which is shared with the terrestrial microwave backhaul links. We focus on the maximization of the network throughput considering a penalizing term to restrict the use of the satellite links in order to avoid the expensive cost of satellite bandwidth. The interference resulting from the spectrum sharing assumption makes the joint power and flow assignment a very challenging problem. We propose a convex relaxation approach which eases the formulation and allows the implementation of efficiency convex optimization tools to achieve a feasible solution to the original problem. Supporting results based on numerical simulations validate the proposed approach.
\end{abstract}

\section{INTRODUCTION}

The upcoming fifth generation of cellular systems (5G) deployment have posed numerous challenges, mainly in terms of supporting very high data rates with low end-to-end delays [1], [2]. Accomplishment of these goals while keeping good balance between spectral and energy efficiency has been the focus of many research works. One of the major challenges for the $5 \mathrm{G}$ deployment is to substantially improve the underlying backhaul infrastructure [2]-[5].

In this paper, we focus on wireless backhaul networks due to its implementation flexibility and cost efficiency. Current wireless backhaul network operates on the microwave spectrum, i.e. 6-60 GHz but rarely going over $30 \mathrm{GHz}$. At these frequencies, the signal attenuation is quite high resulting in short-range links (few tens of kilometers). Therefore, either using mm-Wave or microwave bands, multiple hops are required to overcome long distances and/or obstacles in the propagation path [6], [7].

In order to improve the capacity of mobile wireless backhaul networks, the concept of a seamlessly Integrated SatelliteTerrestrial Backhaul Network (ISTB) capable of jointly exploiting the terrestrial and satellite links depending on the traffic demands has been recently proposed [8], [9]. In order to improve the spectrum efficiency of such networks, aggressive frequency reuse in the Ka band between terrestrial and satellite links have been investigated in [10], [11]. The centralized management of the ISTB network is possible thanks to the emerging network softwarization, i.e. Software Defined Network (SDN) applied to 5G [12]. In this paper, we focus on such ISTB multi-hop wireless network capable of jointly exploiting the terrestrial and satellite links depending on the traffic demands. In this case, the resulting interference needs to be taken into account in order to guarantee operation of the overall backhaul network.

Flow assignment for wireless multi-hop mesh networks have been an attractive area of research during the last years [13], [14]. The amount of intermediate nodes between the source and the destination results in many optional paths, making the flow assignment decision non-trivial. Several metrics have been proposed to determine the best path to reach a destination. The shortest path metric is one of the most popular ones, which selects the path with least number of hops between source and destination. However, the later does not take into account the link capacity and may lead to poor network performance. Alternatively, other protocols aim at obtaining high throughput end-to-end path [15]. In the ISTB network considered herein, the quality of the links may suffer from interference of nearby links operating in the same frequency band. In this context, link capacity is usually considered as the metric to quantify the quality of the link, which captures the interference effect [16], [17]. In this context, smart Radio Resource Management (RRM) comes into play, since the interference level depends on how the radio resources are allocated. Therefore, in this paper, we propose a novel holistic optimization in order to coordinate the flow assignment and the management of radio resources such as the transmit power.

Joint flow and radio resource assignment have been previously considered in [6], [18]-[23]. The works in [6], [18][20], [22] have focused on general terrestrial wireless backhaul networks. [6] assumes the availability of antenna arrays capable of performing both transmit and receive beamforming to mitigate the interference. [22] extends the work of [19] to deal with interference in Code-Division Multiple Access (CDMA) wireless networks. CDMA is also considered in [18], where the main goal is to minimize the total power. [20] proposes a new approach which makes flow assignment and resource allocation decisions based on node location information solely. The work in [21] considers the ITSB network but without spectrum sharing, i.e. without interference, while the work in [23] does consider spectrum sharing but targets the frequency allocation and flow scheduling.

This paper differs from the previous works in three aspects. 
First, spectral reuse is considered among the ITSB network which results in an interference environment that significantly differs from the CDMA case. Second, our goal is to find the optimal holistic strategy that maximizes the sum throughput of the network, while restricting the use of the satellite link whenever possible in order to avoid the expensive cost of satellite bandwidth. Third, we focus on the joint power and flow assignment for a given wireless network topology and end-to-end traffic demands.

The reminder of this paper is organized as follows. Section II introduces the system model and the associated interference environment. After that, Section III presents the proposed joint power and flow assignment problem formulation followed by the proposed solution in Section IV. Supporting numerical results are provided in Section V, and Section VI states the conclusion.

\section{SySTEM MODEL}

We consider a mesh backhaul topolgy composed of $N$ nodes and $L$ links, which we model as a bi-directed graph $(\mathcal{N}, \mathcal{L})$, where $\mathcal{N}$ is the set of nodes (including terrestrial, hybrid and satellite nodes) and $\mathcal{L}$ is the set of the directed wireless links (including terrestrial links, Space-to-Earth and Earth-to-Space links).

Let $\mathcal{T}$ denote the set of links that are terrestrial, i.e. emitted and received by terrestrial transceivers, with $|\mathcal{T}|=T$, being | $\mid$ the cardinality of the set. Similarly, let $\mathcal{S}$ be the set of satellite links, i.e. emitted or received by the satellite, being $|\mathcal{S}|=S$. The set $\mathcal{S}$ is composed of the union of two orthogonal sets, namely $\mathcal{S}=\mathcal{S}_{D L} \cup \mathcal{S}_{U L}$, where $\mathcal{S}_{D L}$ and $\mathcal{S}_{U L}$ refer to the "Space-to-Earth" and the "Earth-to-Space" satellite links, respectively. The terrestrial part of the graph is bi-directed, meaning that for each pair of nodes, there are two links that transmit in opposite directions; while the satellite part of the graph composed on the $\mathcal{S}$ links is directed, i.e. there is a single link between the corresponding nodes. For each $\ell \in \mathcal{L}$, we define the vector $\mathbf{a}_{\ell} \in \mathbb{R}^{N}$ whose entries are defined as,

$$
a_{\ell}(n)= \begin{cases}-1 & \text { if } \ell \in \mathcal{O}(n), \\ 1 & \text { if } \ell \in \mathcal{I}(n), \\ 0 & \text { if otherwise }\end{cases}
$$

where $\mathcal{O}(n)$ denotes the set of links outgoing from node $n$, and $\mathcal{I}(n)$ denotes the set of links incoming to node $n$. The network topology can be described in a compact manner by using the node-link incidence matrix $\mathbf{A} \in \mathbb{R}^{N \times L}$ defined as, $\mathbf{A}=\left[\begin{array}{llll}a_{1} & a_{2} & \ldots & a_{L}\end{array}\right]$

In order to model the data flow in the network, we define the source vector $\mathbf{s}^{(d)} \in \mathbb{R}^{N}$ whose entry $s^{(d)}(n)$ denotes the non-negative flow injected into the network at node $n, n \neq d$ and intended for node $d$. The component $s^{(d)}(d)$ is defined as $-\sum_{n \neq d} s^{(d)}(n)$ so that the flow conservation law is satisfied. Furthermore, we define the flow vector $\mathbf{x}^{(d)} \in \mathbb{R}_{+}^{L}$ as the vector with elements $x^{(d)}(\ell)$ containing the amount of flow in link $\ell$ destined to node $d$. Using the previous definition, the flow conservation low can be conveniently reduced to the following compact expression,

$$
\mathbf{A} \mathbf{x}^{(d)}=\mathbf{s}^{(d)}, \text { for } d=1, \ldots, D .
$$

According to (2), each link $\ell$ has to support the following amount of traffic $t_{\ell}=\sum_{d=1}^{D} x^{(d)}(\ell)$. General flow assignment techniques assume that the capacity of the link is high enough to carry such amount of traffic. However, the link capacity $c_{\ell}$ strongly depends on the radio resources allocated to that link, e.g. the operating frequency, the bandwidth, the transmit power, etc, and is generally expressed as,

$$
c_{\ell}\left(\mathrm{r}_{\ell}\right)=\ln \left(1+\operatorname{SINR}_{\ell}\left(\mathrm{r}_{\ell}\right)\right)
$$

where $\operatorname{SINR}_{\ell}\left(\mathrm{r}_{\ell}\right)$ denotes the Signal-to-Interference plus Noise Ratio of the $\ell$-th link and $r_{\ell}$ represents the radio resources assigned to link $\ell$. Therefore, the capacity constraints $t_{\ell} \leq$ $c_{\ell}\left(\mathrm{r}_{\ell}\right), \ell=1, \ldots, L$, should be satisfied. In the next section, we provide the formulation of $\operatorname{SINR}_{\ell}\left(\mathrm{r}_{\ell}\right)$ for the terrestrial and satellite links of the considered network.

\section{A. Interference Model}

In the proposed satellite-terrestrial spectrum sharing scenario, the radio resource to be allocated is $r_{\ell}=\left\{P_{\ell}\right\}$. For the interference model, we differentiate between two types of receivers:

1) Terrestrial Receiver: The terrestrial receivers collect two types of interference, which are listed below:

- Terrestrial-to-terrestrial: Aggressive frequency reuse among terrestrial backhaul links is assumed targeting a spectral efficiency improvement due to the frequency re-use.

- Satellite-to-terrestrial: Due to the spectrum sharing of the terrestrial links and the satellite Earth-toSpace links in the $27.5-29.5 \mathrm{GHz}$ band, interference from satellite backhauling terminals to the terrestrial receivers shall be considered.

As a consequence, the interference power seen by the link $\ell \in \mathcal{T}$ is the sum of the power received from the terrestrial emitters and neighboring satellite terminals operating in the same carrier frequency. Therefore, the corresponding SINR for the $\ell, \ell \in \mathcal{T}$, operating at carrier frequency $k$ is given by,

$$
\begin{aligned}
& \operatorname{SINR}_{\ell}\left(\mathrm{r}_{\ell}\right)= \\
& \frac{P_{\ell} \cdot H_{\ell, \ell, k}}{\sum_{\substack{i \in \mathcal{T}(k) \\
i \neq \ell}} P_{i} \cdot H_{\ell, i, k}+\sum_{j \in \mathcal{S}_{U P}(k)} P_{j} \cdot H_{\ell, j, k}+\sigma_{\ell}^{2}}, \\
& \ell \in \mathcal{T}
\end{aligned}
$$

where $\mathcal{T}(k)$ and $\mathcal{S}_{U P}(k)$ denote the set of terrestrial and satellite "Earth-to-Space" links operating at frequency carrier $k, P_{x}$ is the transmit power of the $x$-th link' transmit station, $H_{\ell, x, y}$ denotes the channel coefficient between the $x$-th link' transmit node and the $y$-th link' receive node, respectively, for a certain frequency $k$, and $\sigma_{\ell}^{2}$ is the noise power at the $\ell$-th link' receiver, which in this case is a terrestrial receiver.

2) Satellite Receiver: Due to the spectrum sharing of the terrestrial links and the satellite Space-to-Earth links in the $17.7-19.7 \mathrm{GHz}$ band, interference from terrestrial 
backhauling transmitters to the satellite backhauling terminals shall be considered.

In particular, we can formulate the SINR of the $\ell$-th link, $\ell \in \mathcal{S}_{D L}$, operating at the $k$-th carrier as,

$\operatorname{SINR}_{\ell}\left(\mathrm{r}_{\ell}\right)=\frac{P_{\ell} \cdot H_{\ell, \ell, k}}{\sum_{i \in \mathcal{T}(\ell)} P_{i} \cdot H_{\ell, i, k}+I_{\mathrm{co}, \ell}+\sigma_{\ell}^{2}}, \ell \in \mathcal{S}_{D L}$

where $\mathcal{T}(k), P_{x}, H_{\ell, \ell, k}$ and $H_{\ell, i, k}$ follow the same definition as in (4). Note that $P_{\ell}$ for $\ell \in \mathcal{S}_{D L}$ denotes the satellite transmit power, and $\sigma_{\ell}^{2}$ is the noise power, in this case seen at the satellite dish antenna. The term $I_{\mathrm{co}, \ell}$ accounts for the interference received from adjacent satellite beams, which is very common in multi-beam satellite systems.

Note that the interference from the satellite to the terrestrial receivers and from the terrestrial receivers to the satellite is neglected due to the huge propagation distance, the limitation in the maximum EIRP density of the current Ka band satellite system and the strong directivity of the terrestrial backhaul antennas.

\section{Problem Formulation}

Our goal is to find the optimal power and flow assignment that maximizes the sum throughput of the network, i.e. $\sum_{d=1}^{D} \sum_{\substack{n=1 \\ n \neq d}}^{N}\left(s^{(d)}(n)\right)$, and at the same time account for the cost of using the satellite links. According to the previous definitions, the holistic optimization of flow assignment and radio resources is formulated as follows,

$$
\begin{array}{ll}
\max _{\{\mathbf{x}, \mathbf{r}\}} & \sum_{\substack{d=1 \\
\text { s.t. }}}^{D} \sum_{\substack{n=1 \\
n \neq d}}^{N} s^{(d)}(n)-\lambda \sum_{d=1}^{D} \sum_{\ell \in \mathcal{S}} x^{(d)}(\ell) \\
& \mathbf{A x} \mathbf{x}^{(d)}=\mathbf{s}^{(d)}, \quad d=1, \ldots, D \\
& \mathbf{x}^{(d)} \succcurlyeq 0, \quad d=1, \ldots, D \\
& t_{\ell}=\sum_{d=1}^{D} x^{(d)}(\ell), \quad \ell=1, \ldots, L \\
& t_{\ell} \leq c_{\ell}\left(\mathrm{r}_{\ell}\right), \quad \ell=1, \ldots, L \\
& 0 \leq P_{\ell} \leq P_{\ell}^{\max }, \quad \ell=1, \ldots, L
\end{array}
$$

where $\{\mathbf{x}, \mathrm{r}\}$ denote the optimization variables, namely the data flows $\mathbf{x}^{(1)}, \ldots, \mathbf{x}^{(D)}$ and the radio resource variables, in this case $P_{1}, \ldots, P_{L}$. The second term of the objective function in (6) is composed of $\lambda \in \mathbb{R}_{+}$multiplied by the sum of flows on the satellite links. The transmit power per link is limited to $P_{\ell}^{\max }$. The capacity constraints in (6) are not jointly convex due to presence of interference in $c_{\ell}\left(\mathrm{r}_{\ell}\right)$. Therefore, finding the global optimal solution is very challenging.

\section{Proposed Flow and Radio Resources ASSIGNMENT}

Similar to [22], we make use of the following approximation to relax the capacity constraints,

$$
c_{\ell}\left(\mathrm{r}_{\ell}\right)=\ln \left(1+\operatorname{SINR}_{\ell}\left(\mathrm{r}_{\ell}\right)\right) \approx \ln \left(\operatorname{SINR}_{\ell}\left(\mathrm{r}_{\ell}\right)\right),
$$

which is satisfied in general for $\operatorname{SINR}_{\ell}\left(\mathrm{r}_{\ell}\right)>5$. The later can be achieved with proper carrier allocation such the one proposed in [11]. We can rewrite the capacity $c_{\ell}\left(\mathrm{r}_{\ell}\right)$ as follows:

1) For the terrestrial links: For the terrestrial links $\ell \in \mathcal{T}$, using (4) and (7), we obtain the following,

$$
\begin{array}{r}
c_{\ell}\left(\mathrm{r}_{\ell}\right) \approx \\
-\ln \left(\sum_{\substack{i \in \mathcal{T}(k) \\
i \neq \ell}} \frac{H_{\ell, i, k}}{H_{\ell, \ell, k}} P_{i} P_{\ell}^{-1}+\sum_{j \in \mathcal{S}_{U P}(k)} \frac{H_{\ell, j, k}}{H_{\ell, \ell, k}} P_{j} P_{\ell}^{-1}\right. \\
\left.+\frac{\sigma_{\ell}^{2}}{H_{\ell, \ell, k}} P_{\ell}^{-1}\right)
\end{array}
$$

We apply the change of variable $P_{x}=\exp \left(Q_{x}\right)$ to (8) and obtain the following expression,

$$
\begin{array}{r}
c_{\ell}\left(\mathrm{r}_{\ell}\right) \approx \\
-\ln \left(\sum_{\substack{i \in \mathcal{T}(k) \\
i \neq \ell}} \frac{H_{\ell, i, k}}{H_{\ell, \ell, k}} e^{Q_{i}-Q_{\ell}}+\sum_{j \in \mathcal{S}_{U P}(k)} \frac{H_{\ell, j, k}}{H_{\ell, \ell, k}} e^{Q_{j}-Q_{\ell}}\right. \\
\left.+\frac{\sigma_{\ell}^{2}}{H_{\ell, \ell, k}} e^{-Q_{\ell}}\right),
\end{array}
$$

which is concave in variable $Q$.

2) For the satellite links: Similarly, for $\ell \in \mathcal{S}_{D L}$ we make use of (5) combined with (7) and obtain the following expression,

$$
\begin{aligned}
& c_{\ell}\left(\mathrm{r}_{\ell}\right) \approx \\
& \quad-\ln \left(\sum_{i \in \mathcal{T}(\ell)} \frac{H_{\ell, i, k}}{H_{\ell, i, k}} P_{i} P_{\ell}^{-1}+\frac{I_{\mathrm{co}, \ell}+\sigma_{\ell}^{2}}{H_{\ell, \ell, k}} P_{\ell}^{-1}\right) .
\end{aligned}
$$

Applying the change of variable $P_{x}=\exp \left(Q_{x}\right)$ to (10), we obtain the following concave expression,

$$
\begin{aligned}
c_{\ell}\left(\mathrm{r}_{\ell}\right) & \approx \\
& -\ln \left(\sum_{i \in \mathcal{T}(\ell)} \frac{H_{\ell, i, k}}{H_{\ell, i, k}} e^{Q_{i}-Q_{\ell}}+\frac{I_{\mathrm{co}, \ell}+\sigma_{\ell}^{2}}{H_{\ell, \ell, k}} e^{-Q_{\ell}}\right) .
\end{aligned}
$$

By assuming the approximations proposed in (9) and (11), the problem (6) is convexified. Note that the constraint on the transmit power $0 \leq P_{\ell} \leq P_{\ell}^{\max }$ translates into $0 \leq e^{Q_{\ell}} \leq P_{\ell}^{\max }$ which remains strictly convex in the new variable $Q_{\ell}$. As a consequence, a globally optimal solution can be efficiently obtained for the proposed approximate flow and power assignment problem, for instance with the popular Interior Point or Barrier methods [24].

\section{Simulation Results}

For the performance evaluation, we consider the network topology illustrated in Fig. 1, which represent a wireless backhaul topology consisting of $N=7$ nodes, from which node number 7 is the satellite, and $L=18$ directed links. The topology at a hand includes 2 nodes with hybrid terrestrialsatellite transmission capabilities, which are node 1 and 6 . For the sake of simplicity, we assume a single traffic flow, which goes from node 1 to node 6 . In addition, we assume that the terrestrial topology operates using an aggressive frequency reuse pattern with 3 carriers of $56 \mathrm{MHz}$ each. The carrier 


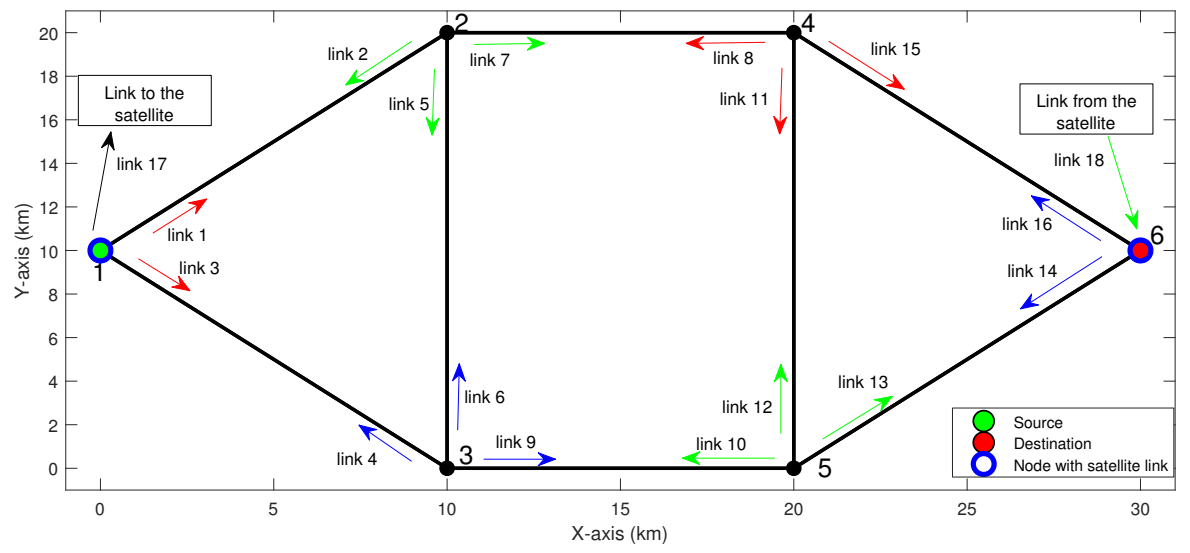

Fig. 1: Topology under evaluation including the frequency allocation represented by red, blue and green arrows.

TABLE I: Simulation Parameters

\begin{tabular}{|c|c|}
\hline \multicolumn{2}{|c|}{ Terrestrial Segment } \\
\hline Parameter & Value \\
\hline No. available carriers & 3 \\
\hline Bandwidth & $56 \mathrm{MHz}$ \\
\hline Spanning frequency & carrier 1 from 17700 to $17756 \mathrm{MHz}$ \\
\hline & carrier 2 from 17756 to $17812 \mathrm{MHz}$ \\
\hline Antenna pattern & $\begin{array}{c}\text { carrier } 3 \text { from } 17812 \text { to } 17868 \mathrm{MHz} \\
\text { ITU-R F.1245-2 }\end{array}$ \\
\hline Max. antenna gain & $24.5 \mathrm{dBi}$ \\
\hline Max. transmit power & $-20.6481 \mathrm{dBW}$ \\
\hline Channel & LoS channel (path loss) \\
\hline Noise power & $-121.52 \mathrm{dBW}$ \\
\hline Antenna height & Between 13 and $48 \mathrm{~m}$ \\
\hline \multicolumn{2}{|c|}{ Satellite Segment } \\
\hline Parameter & Value \\
\hline No. available carriers & 2 \\
\hline Bandwidth & $56 \mathrm{MHz}$ \\
\hline Spanning frequency & $\begin{array}{l}\text { Downlink from } 17812 \text { to } 17868 \mathrm{MHz} \\
\text { Uplink from } 29500 \text { to } 29556 \mathrm{MHz}\end{array}$ \\
\hline Satellite location & $13^{\circ} \mathrm{E}$ \\
\hline Satellite gain & $55.7693 \mathrm{dBi}$ \\
\hline Dish antenna pattern & ITU-R S.465 \\
\hline Max. dish antenna gain & $42.1 \mathrm{dBi}(90 \mathrm{~cm}$ dish $)$ \\
\hline Co-channel interference & $-113 \mathrm{dBW}$ \\
\hline Max transmit power from Earth & $7.9 \mathrm{dBW}$ \\
\hline Max transmit power from Satellite & $9.23 \mathrm{dBW}$ \\
\hline Channel & LoS channel (path loss) \\
\hline Link distance & $35.786 \mathrm{~km}$ \\
\hline Noise power & $-126.94 \mathrm{dBW}$ \\
\hline Dish antenna height & Between 13 and $48 \mathrm{~m}$ \\
\hline
\end{tabular}

allocation is depicted in Fig. 1 with red, blue and green arrows, each color representing a different carrier. Note that the satellite is reusing one of these carriers for the downlink, while the uplink is assumed to be in an interference-free carrier for simplicity. A summary of the system parameters considered for the simulation set-up and for the channel coefficients generation is given in Table I.

Fig. 2 shows the channel matrix instance for the topology depicted in Fig. 1, where the diagonal elements correspond to the intended links, while the off-diagonal elements correspond to non-desired interference links. It can be observed that due to the aggressive frequency reuse, many non-diagonal elements are significantly high.

Fig. 3 and Fig. 4 illustrate the amount of traffic flow that is routed through the satellite segment and the overall throughput delivered at the destination node 7 , respectively, as a function of the penalty $\lambda$ assigned to the satellite links so as to account

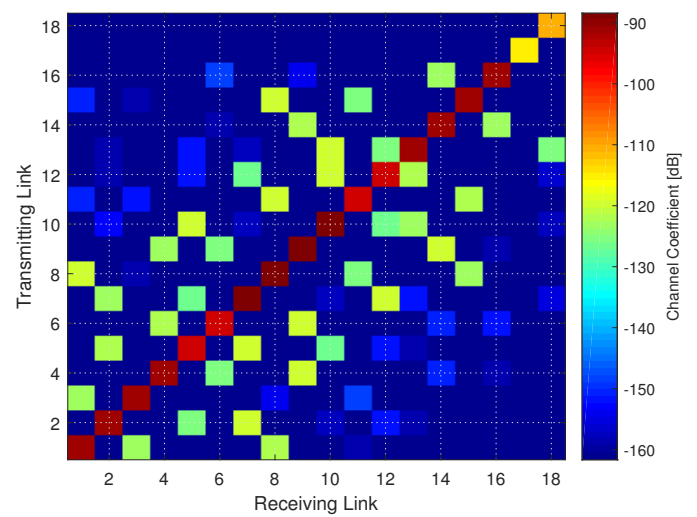

Fig. 2: Channel coefficients for the assumed frequency assignment

for the cost of using such links. It can be observed that when the cost of using the satellite links is high, all the traffic is routed via the terrestrial network, achieving a total throughput of $4.329 \mathrm{~b} / \mathrm{s} / \mathrm{Hz}$. If the cost of using the satellite is reduced, then the satellite links come into play and support the terrestrial links to deliver the traffic to the destination. The maximum throughput is achieved at $\lambda=0.49$ where the total throughput at node 7 is $6.891 \mathrm{~b} / \mathrm{s} / \mathrm{Hz}$, which represents a $60 \%$ increase with respect to the terrestrial-only counterpart.

Focusing on $\lambda=0.49$, Fig. 5 illustrated in a schematic way the flow assignment per each link according to the proposed approach, where $2.61 \mathrm{~b} / \mathrm{s} / \mathrm{Hz}$ are sent through the satellite and $4.33 \mathrm{~b} / \mathrm{s} / \mathrm{Hz}$ are routed via the terrestrial infrastructure. For the same scenario, Table II shows the assigned power which is compared to the maximum available power. It can be observed that in some links the transmit power is reduced so as to not caused harmful interference to links that are important for the traffic delivery. For instance, links 12 and 13 share the same carrier but link 13 is fundamental for the connection with the destination node 7 . Therefore, link 13 is given the maximum power while link 12 is assigned a fraction of the maximum available power. 


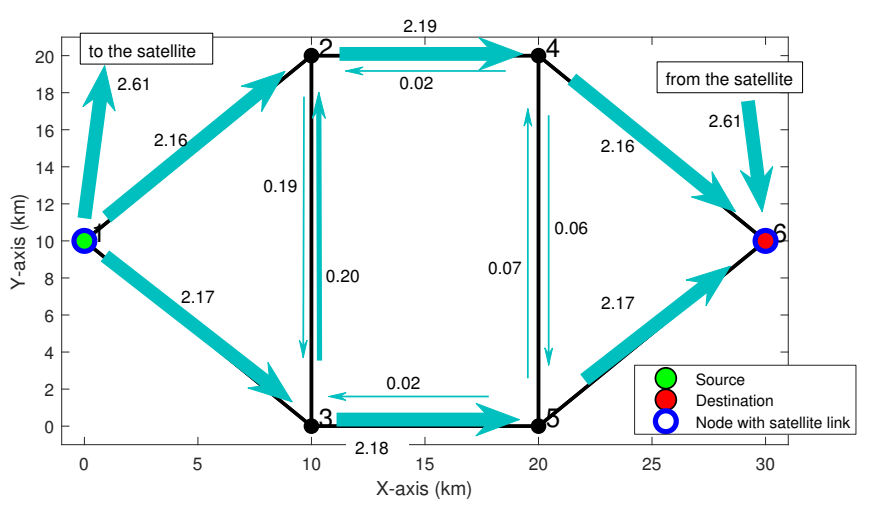

Fig. 5: Flow assignment provided by the proposed algorithm with $\lambda=0.49$

TABLE II: Power Assignment Results for $\lambda=0.49$

\begin{tabular}{|c|c|c|}
\hline & Used Power [dB] & Max Available Power [dB] \\
\hline link 1 & -20.6481 & -20.6481 \\
link 2 & -22.7698 & -20.6481 \\
link 3 & -20.6481 & -20.6481 \\
link 4 & -22.4787 & -20.6481 \\
link 5 & -21.6277 & -20.6481 \\
link 6 & -21.5622 & -20.6481 \\
link 7 & -21.0660 & -20.6481 \\
link 8 & -32.2044 & -20.6481 \\
link 9 & -21.0214 & -20.6481 \\
link 10 & -31.9947 & -20.6481 \\
link 11 & -26.8576 & -20.6481 \\
link 12 & -26.7015 & -20.6481 \\
link 13 & -20.6481 & -20.6481 \\
link 14 & -22.3586 & -20.6481 \\
link 15 & -20.6481 & -20.6481 \\
link 16 & -22.3871 & -20.6481 \\
link 17 & 7.9 & 7.9 \\
link 18 & 9.2286 & 9.2307 \\
\hline
\end{tabular}

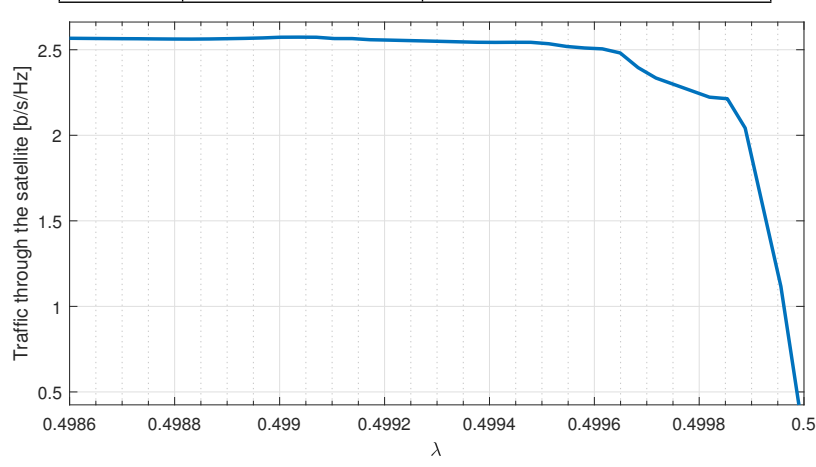

Fig. 3: Traffic flow routed through the satellite as a function of $\lambda$

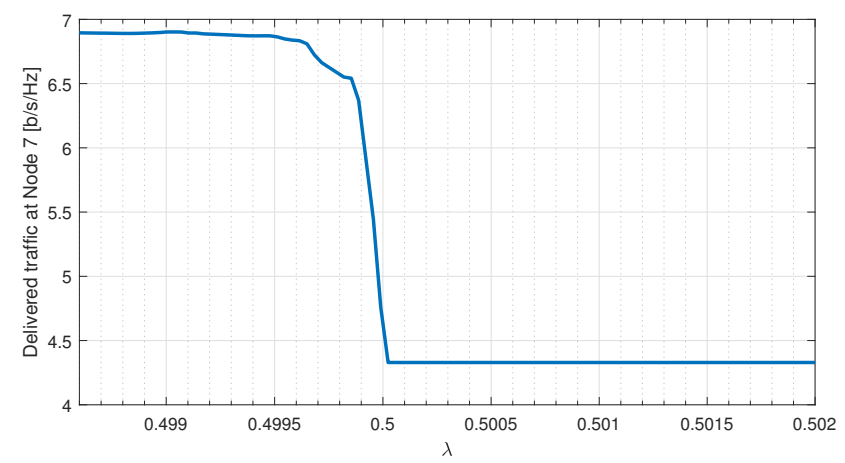

\section{CONCLUSION}

In this paper, we presented a novel joint power and flow assignment algorithm for integrated satellite-terrestrial networks with aggressive frequency reuse and spectral coexistence between terrestrial and satellite segments. The problem is formulated by considering a penalty term to account for the use of the satellite links only in cases where the terrestrial segment is not able to absorb the traffic to be delivered. Given the intractability of the problem, we proposed an alternative approximation to relax the capacity constraint which renders a strictly convex problem. Results based on numerical simulations were presented, which showed that the concept of integrated satellite-terrestrial backhaul network can effectively work under the spectral coexistence assumption by considering the proposed power and flow assignment approach. In future works, the joint frequency, power and flow assignment would be investigated.

\section{ACKNOWLEDGMENT}

This work was partially supported by the Luxembourg National Research Fund (FNR) under the CORE project "ROSETTA" (11632107), the CORE project "ProCAST", and the ANR-FNR project "SIERRA" - Spectral efficient receivers and resource allocation for cognitive satellite communications.

\section{REFERENCES}

[1] A. Gupta and R.K. Jha, "A Survey of 5G Network: Architecture and Emerging Technologies," IEEE Access, vol. 3, pp. 1206-1232, Aug. 2015.

[2] X. Ge, H. Cheng, M. Guizani, and T. Han, "5G Wireless Backhaul Networks: Challenges and Research Advances," IEEE Network, vol. 28, no. 6, pp. 6-11, Dec. 2014.

[3] H.S. Dhillon and G. Caire, "Wireless Backhaul Networks: Capacity Bounds, Scalability Analysis and Design Guidelines," IEEE Trans. Wireless Commun., vol. 14, no. 11, pp. 6043-6056, Jun. 2015.

[4] E. Hossain and M. Hasan, "5G Cellular: Key Enabling Technologies and Research Challenges," IEEE Instrum. Meas. Mag., vol. 18, no. 3, pp. 11-21, Jun. 2015.

[5] U. Siddique, H. Tabassum, E. Hossain, and D.I. Kim, "Wireless Backhauling of 5G Small Cells: Challenges and Solution Approaches," IEEE Trans. Wireless Commun., vol. 22, no. 5, pp. 22-31, Oct. 2015.

[6] M. Cao, X. Wang, S.J. Kim, and M. Madihian, "Multi-Hop Wireless Backhaul Networks: A Cross-Layer Design Paradigm," IEEE J. Sel. Areas Commun., vol. 25, no. 4, pp. 738-748, May 2007.

[7] M.P. Anastasopoulos, P.D.M. Arapoglou, R. Kannan, and P.G. Cottis, "Adaptive Routing Strategies in IEEE 802.16 Multi-Hop Wireless Backhaul Networks Based On Evolutionary Game Theory," IEEE J. Sel. Areas Commun., vol. 26, no. 7, pp. 1218-1225, Sept. 2008.

[8] European Union's Horizon 2020 research and innovation programme, "SANSA - Shared Access Terrestrial-Satellite Backhaul Network enabled by Smart Antennas," http://sansa-h2020.eu/.

[9] F. Mendoza, R. Ferrús, and O. Sallent, "Flexible Capacity and Traffic Management for Hybrid Satellite-Terrestrial Mobile Backhauling Networks," International Symposium on Wireless Communication Systems (ISWCS), Poznan, Poland, Sept. 2016.

[10] X. Artiga et al., "Spectrum Sharing in Hybrid Terrestrial-Satellite Backhaul Networks in the Ka-Band," European Conference on Networks and Communications (EUCnC), Oulu, Finland, Jun. 2017. 
[11] E. Lagunas, S. Maleki, L. Lei, C. Tsinos, S. Chatzinotas, and B. Ottersten, "Carrier Allocation for Hybrid Satellite-Terrestrial Backhaul Networks," ICC Workshop on Satellite Communications: Challenges and Integration in the 5G ecosystem, Paris, France, May 2017.

[12] M. Corici et al., "SATis5: A 5G Testbed Integrating Satellite and Terrestrial Infrastructures," Advanced Satellite Multimedia Systems Conference (ASMS), Berlin, Germany, Sept. 2018.

[13] A.H. Sherin, H.S. Hassanein, and G. Takahara, Routing for Wireless Multi-Hop Networks, Springer-Verlag, New York, USA, 2013.

[14] T. Meng, F. Wu, Z. Yang, G. Chen, and A.V. Vasilakos, "Spatia Reusability-Aware Routing in Multi-Hop Wireless Networks," IEEE Trans. Comput., vol. 65, no. 1, pp. 244-255, Jan. 2016.

[15] J. Nuñez Martínez and J. Mangues, "A Survey on Routing Protocols that really exploit Wireless Mesh Networks," Journal of Communications, Academy Publisher, vol. 5, no. 3, pp. 211-231, Mar. 2010.

[16] G. Parassidis, "Interference-Aware Routing in Wireless Multihop Networks," PhD, ETH Zurich, Switzerland, 2008.

[17] Q. Zhu, Z. Yuan, J. Song, Z. Han, and T. Basar, "Interference Aware Routing Game for Cognitive Radio Multi-Hop Networks," IEEE J. Sel. Areas Commun., vol. 30, no. 10, pp. 2006-2015, Nov. 2012.

[18] P. Moberg, "Simultaneous Routing and Resource Allocation in Multihop
Wireless Networks using Optimization," IEEE Global Telecommun. Conf. (GLOBECOM), San Francisco, CA, USA, Nov. 2006.

[19] L. Xiao, M. Johansson, and S.P. Boyd, "Simultaneous Routing and Resource Allocation via Dual Decomposition," IEEE Trans. Commun., vol. 52, no. 7, pp. 1136-1144, Jul. 2014.

[20] R. Di Taranto and H. Wymeersch, "Simultaneous Routing and Power Allocation using Location Information," ASILOMAR Conf. in Signals, Systems and Computers, Pacific Grove, CA, USA, Nov. 2013.

[21] L. Rosati and G. Reali, "Jointly Optimal Routing and Resource Allocation in Hybrid Satellite/Terrestrial Networks," Int. Workshop in Satellite and Space Communications, Madrid, Spain, Sept. 2006.

[22] M. Johansson, L. Xiao, and S.P. Boyd, "Simultaneous Routing and Power Allocation in CDMA Wireless Data Networks," IEEE Conf. on Commun. (ICC), Anchorage, AK, USA, May 2003.

[23] M. Shaat, A. Pérez-Neira, G. Femenias, and F. Riera-Palou, "Joint frequency assignment and flow control for hybrid terrestrial-satellite backhauling networks," International Symposium on Wireless Communication Systems (ISWCS), Bologna, Italy, Aug. 2017.

[24] S. Boyd and L. Vandenberghe, Convex Optimization, Cambridge University Press, 2004. 